\title{
Indian minister denies plan for nurse practitioners to fill doctor gap
}

The headline of this News item (BMJ 2015;350:h2789, doi:10. 1136/bmj.h2789) incorrectly refers to Arun Kumar Panda, additional secretary at the Ministry of Health and Family

Welfare, as a "minister." We apologise for this error. The headline should have read: "Indian health official denies plan for nurse practitioners to fill doctors gap"

Cite this as: BMJ 2015;350:h2923

๑ BMJ Publishing Group Ltd 2015 